\title{
MITRAL-AORTIC INTERVALVULAR FIBROSA PSEUDOANEURYSM
}

\author{
MARIA BONOU, MD, PHD ${ }^{1}$, EVA D PAPADIMITRAKI, MD, PHD ${ }^{1}$, SOPHIA VAINA, MD, PHD², \\ GLAFKOS KELEPESHIS, MD ${ }^{1}$, KOSTAS TSAKALIS, MD ${ }^{1}$, NIKOLAOS ALEXOPOULOS, MD, PHD ${ }^{3}$, AND \\ JOHN BARBETSEAS, MD, PHD ${ }^{1}$ \\ 'DEPARTMENT OF CARDIOLOGY, LAIKO GENERAL HOSPITAL, ATHENS, GREECE \\ ${ }^{2}$ FIRST DEPARTMENT OF CARDIOLOGY, HIPPOKRATION HOSPITAL, UNIVERSITY OF ATHENS, ATHENS, GREECE \\ ${ }^{3}$ DEPARTMENT OF CARDIOLOGY, ATHENS EUROCLINIC, ATHENS, GREECE
}

Pseudoaneurysm of the mitral aortic intervalvular fibrosa (MAIVF-P) usually ensues as a complication of endocarditis or aortic valve surgery. When large, symptomatic or related to complications (rupture, compression of adjacent structures, embolic events, mitral regurgitation or heart failure) it warrants surgical excision. The natural course of uncomplicated/asymptomatic MAIVFPs is largely unknown since most patients are offered surgery. Increased surgical risk imposed by repeat operations in the majority of these patients is an important consideration and conservative treatment should not be excluded in selected cases. Herein we present two illustrative cases of MAIVF-P manifesting with significant arrhythmogenesis and complex endocarditis respectively. Both patients were managed conservatively. By briefly reviewing the existing literature, we discuss important diagnostic and therapeutic issues for MAIVF-Ps. To our knowledge complex ventricular arrhythmia has not been previously described as a prominent manifestation of MAIVF-P.

KEY WORDS: Mitral-aortic intervalvular fibrosa pseudoaneurysm · Endocarditis · Pseudoaneurysm • Ventricular arrhythmia.

\section{INTRODUCTION}

Pseudoaneurysm of the mitral aortic intervalvular fibrosa (MAIVF-P) usually ensues as a complication of endocarditis or aortic valve (AV) surgery. The widespread use of transesophageal echocardiography (TEE) and contemporary imaging techniques has enabled prompt diagnosis of this clinical entity, which does not seem to be so rare.

MAIVF-P may cause symptoms or be related to significant complications that warrant timely diagnosis and surgical treatment. Uncomplicated asymptomatic MAIVF-Ps may have a more benign natural history. Herein we present two illustrative cases of MAIVF-P.

\section{CASE}

\section{CASE 1}

A 42-year-old female patient presented with one year history of palpitations and exertional dyspnea. She had under- gone AV replacement (AVR) with a Sorin $19 \mathrm{~mm}$ bileaflet mechanical valve two years prior to presentation, for severe aortic regurgitation (AR) of a bicuspid AV. Coronary angiography at the time of surgery had shown no epicardial coronary artery stenosis whereas one year post surgery, transthoracic echocardiogram (TTE) had shown mild prosthetic valve regurgitation. There was no history of endocarditis. Based on the findings of a 24 hour Holter which had revealed 29\% (29900) premature ventricular contractions (PVCs) and $1.2 \%$ premature supraventricular contractions (PSVCs), previous treating physicians had administered metoprolol and subsequently verapamil, with inadequate symptom control.

On admission her blood pressure was 125/75 mm Hg, saturations were normal and there was no fever. Her electrocardiogram showed sinus rhythm of $66 \mathrm{bpm}$ with multiple PVCs and normal PR, QRS and QT intervals. Twenty four hour Holter recording now showed the presence of $13 \%$ PVCs of multiple morphologies and 3\% PSVCs (Fig. 1A). On TTE

- Received: May 14, 2015 •Revised: September 3, 2015 •Accepted: November 18, 2015

- Address for Correspondence: Eva D Papadimitraki, Department of Cardiology, Laiko General Hospital, 17 Agiou Thoma Street, Athens 11527, Greece

Tel: +30-210-7456258, Fax: +30-213-20161761, E-mail: evapapadimitraki@hotmail.com

- This is an Open Access article distributed under the terms of the Creative Commons Attribution Non-Commercial License (http://creativecommons.org/licenses/by-nc/3.0)

which permits unrestricted non-commercial use, distribution, and reproduction in any medium, provided the original work is properly cited. 

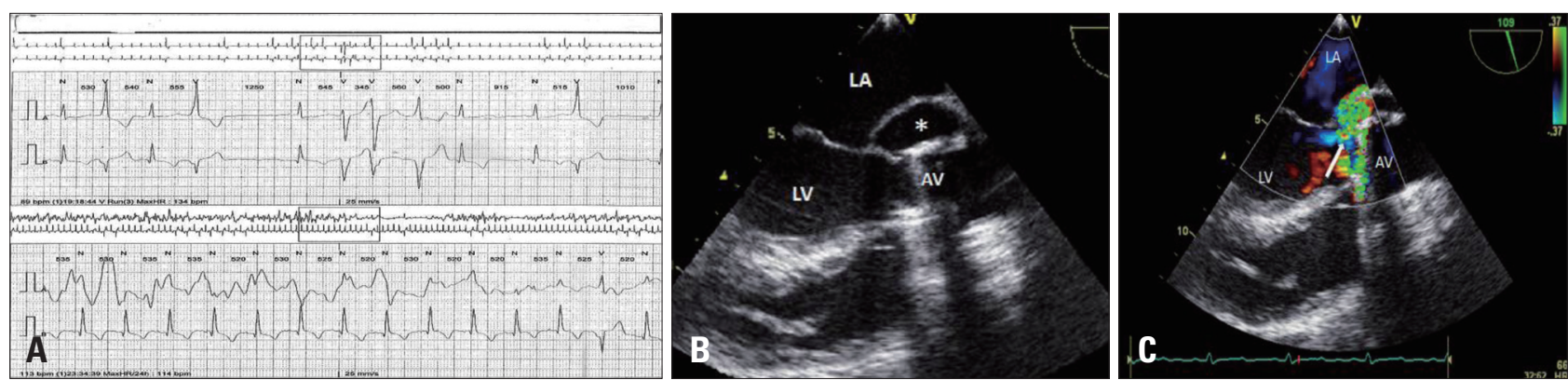

Fig. 1. Significant arrhythmogenesis caused by a mitral-aortic intervalvular fibrosa pseudoaneurysm (MAIVF-P). A: 24-hour Holter recording of the patient in case 1. PVCs of multiple morphologies are depicted. The electrophysiologic study confirmed that the majority of those were of left ventricular outflow tract origin. B: Transesophageal echocardiogram (TEE), long axis view of the aorta systolic frame. A MAIVF-P exhibiting systolic expansion is seen (asterisk). C: TEE, long axis view of the aorta, color Doppler interrogation. The MAIVF-P fills during systole (arrow). AV: aortic valve, LA: left atrium, LV: left ventricle.

biventricular systolic function was normal and again there was only mild prosthetic valve regurgitation. However, TEE revealed the presence of an aneurismal structure measuring approximately $3.3 \mathrm{~cm}$ at its largest dimension posterior to the aortic root and extending to the area of the mitral-aortic intervalvular fibrosa (Fig. 1B and C). The pseudoaneurysmal cavity exhibited systolic expansion and diastolic collapse and communicated with the left ventricular outflow tract (LVOT) through a narrow neck (Supplementary movie 1). This was a MAIVF-P. There were no signs of prosthetic valve dysfunction. Apparently, the reverse blood flow from the pseudoaneurysm into the LVOT during diastole had been misinterpreted as the aforementioned prosthetic valve regurgitation on TTE interrogations. The presence of the MAIVF-P was confirmed by multidedector computed tomography (CT) (Fig. 2). On a subsequent electrophysiologic study (EPS), most of the PVCs were of LVOT origin. Ablation was not performed for safety concerns raised by the proximity of PVC focus to the MAIVFP. Since there had been no history of endocarditis, AV surgery trauma on a substrate of bicuspid aortopathy was felt to be the cause of pseudoaneurysm formation, which apparently triggered both the supraventricular-through its vicinity with the left atrium (LA)-and the ventricular-of LVOT origin-arrhythmia. The patient denied surgical excision and opted for conservative follow-up.

Four years later she presented with worsening dyspnea (New York Heark Association II-III), persistent palpitations and again, the presence of multiple PVCs (19\%). Left ventricular dimensions were normal but the systolic function was now mildly compromised (left ventricular ejection fraction = 45\%). The MAIVF-P diameter remained stable. A coronary angiography showed no major epicardial stenoses or coronary artery compression. On EPS, the LVOT origin of the majority of the PVCs was reconfirmed. Systolic dysfunction was felt to be attributable to the presence of multiple PVCs. ${ }^{1)}$ The patient was still reluctant to surgery.

\section{CASE 2}

A 77-year-old male patient presented with a 10 day history
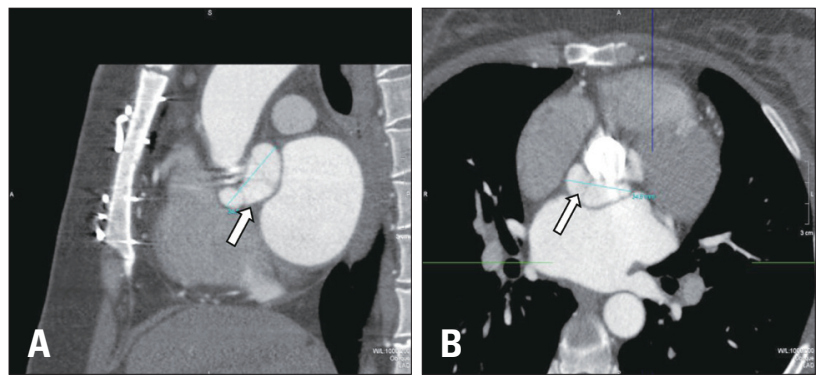

Fig. 2. Multidetector computed tomography showing the mitral-aortic intervalvular fibrosa pseudoaneurysm (arrows) both in the sagittal plane (A) and in the axial plane (B).

of fever. He had undergone combined coronary artery bypass graft surgery and AVR with a bioprosthetic valve for severe aortic stenosis 15 years prior to presentation. Five years before his current admission he had suffered prosthetic valve endocarditis and was re-operated. At that time he had a composite valve graft conduit replacement of the ascending aorta and the prosthesis, with a bioprosthetic valve.

Blood cultures were positive for Gemella morbillorum and an initial TEE interrogation revealed the presence of an AV vegetation. Endocarditis was diagnosed and ceftriaxone with gentamicin were started. Ten days later, the fever still persisted. Asymptomatic complete atrioventricular block with narrow QRS and an escape rhythm of 45 bpm developed, suggesting the presence of an aortic ring abscess that extended into the area of atrioventricular node or the bundle of His. The TEE showed the presence of an anterior annular aortic abscess in the MAIVF area (Fig. 3A, Supplementary movies $2 \mathrm{~A}$ and 3A). Furthermore, vegetations on both, the bioprosthetic AV and the anterior mitral leaflet were now seen (Supplementary movie 2B). Ceftriaxone was replaced by high doses of penicillin G (24000000 U/24 hr). Since the escape rhythm was stable, a temporary pacemaker was not placed. The patient denied reoperation.

Consecutive TEE studies during his hospital stay unveiled the gradual formation of a MAIVF-P. The fever subsided and the heart block reverted to sinus rhythm spontaneously two weeks later. At that time on TEE the perivalvular abscess ap- 

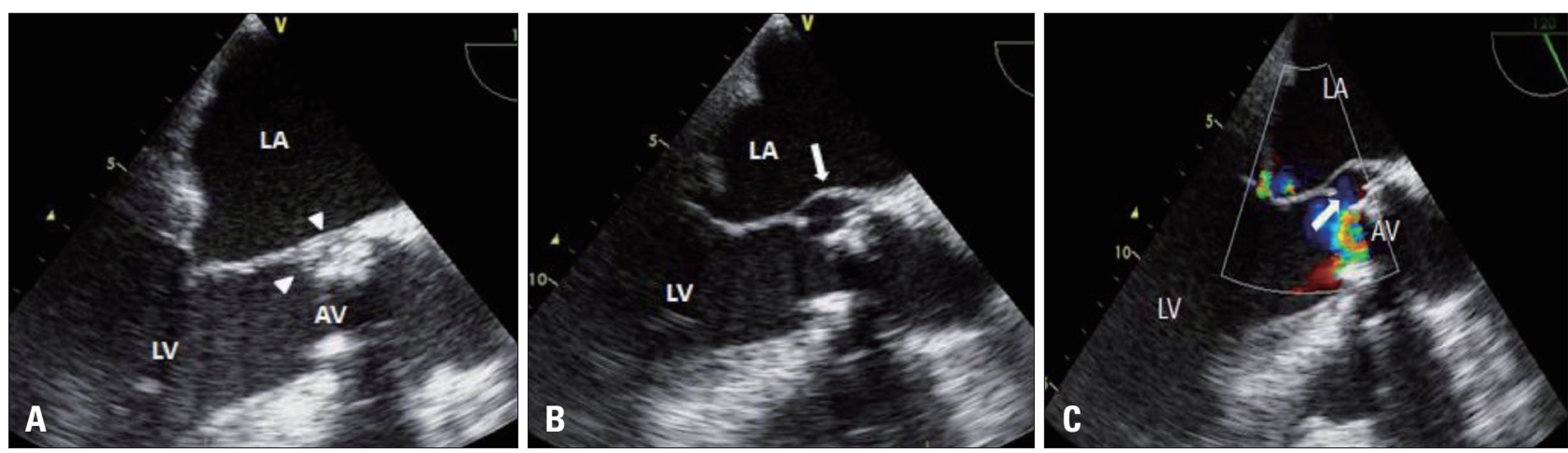

Fig. 3. The gradual formation of a pseidoaneurysm. A: Transesophageal echocardiogram (TEE), long axis view of the aorta. A small echodense space representing an abscess is seen in the mitral-aortic inetrvalvular fibrosa area (arrowheads). B: TEE, long axis view of the aorta, four weeks later. A mitral aortic intervalvular fibrosa pseudoaneurysm (MAIVF-P) at the site of the preexisting abscess is now depicted (arrow). C: TEE, long axis view of the aorta, color Doppler interrogation. The MAIVF-P communicates with the left ventricular outflow tract and fills during systole (arrow). AV: aortic valve, LV: left ventricle, LA: left atrium.

peared to have ruptured into the left ventricle and the initial aortic root thickening gradually had evolved into a small pseudoaneurysm. The MAIVF-P had increased in size, measuring $2.2 \mathrm{~cm}$ four weeks later. A small anterior mitral leaflet perforation leading to mild valvular regurgitation was also depicted (Fig. 3B and C, Supplementary movies 2C-E, 3B and C).

The patient was discharged six weeks after admission. At that time he was afebrile, with mild exertional dyspnea. The inflammatory markers remained low and a 18F-fluorodeoxyglucose positron emission tomography (PET)/CT scan was negative for inflammation. He is currently seen at the Heart Valve Clinic and remains reasonably well.

\section{DISCUSSION}

MAIVF-P is defined as a pseudoaneurysm at the interannular zone between the mitral and AVs, communicating with the LVOT. It was first described by Waldhausen et al. ${ }^{2}$ in 1966. Although until 2009 approximately 90 cases had been presented in medical literature, by 2012 this number had doubled, which implies that MAIVF-P may be not such a rare clinical entity.

\section{AETIOLOGY}

Infection and cardiac surgery are the two most frequent MAIVF-P associations, in line with the two herein presented cases, with infection being the most common cause (78\%). MAIVF is a fibrous structure constituting the continuity between the anterior mitral leaflet and the non coronary cusp of the AV. Its thin and avascular nature makes this region prone to both infection and injury. In fact, MAIVF-P is a wellrecognized complication of native or prosthetic AV endocarditis, where it represents the residue of a spontaneously ruptured or surgically drained abscess cavity into the left ventricle. A MAIVF-P can also result from local trauma after AV surgery with no evidence of infection. AR may contribute to MAIVF$\mathrm{P}$ formation by compromising and/or infecting the MAIVF region through a regurgitant jet. Bicuspid aortopathy is an- other well recognized contributing factor conferring congenital weakness in the MAIVF area. Finally, chest trauma, mitral valve endocarditis, mitral valve surgery related- and congenital cases of MAIVF-P have been reported.

\section{CLINICAL PRESENTATION}

In the series by Sudhakar et al.," MAIVF-P most frequently (39\% of cases) manifested with symptoms and signs of active infection (endocarditis), followed by dyspnea and heart failure (16\% of cases). Cerebrovascular accidents and embolic events are not rare MAIVF-P associations. Chest pain and chest wall masses have also been described whereas up to $10 \%$ of patients with MAIVF-P may be asymptomatic. Palpitations-the presenting complaint of our first patient-is not a common association of MAIVF-P. To our knowledge ventricular arrhythmogenesis has not been previously described as a prominent manifestation of MAIVF-P.

\section{DIAGNOSIS}

The sensitivity of TTE for the diagnosis of MAIVF-P is limited-around $40 \%-$, which is why a low threshold for performing TEE is advocated upon indication. The sensitivity of TEE is approximately $90-95 \%$, and as illustrated in case 2, repeat TEE interrogations may be warranted to identify this complication. The echocardiographic appearance of MAIVF-P is that of a false lumen below the AV annulus at the level of the MAIVF. The pseudoaneurysmal cavity exhibits marked pulsatility with systolic expansion and diastolic collapse and communicates with the LVOT. Color flow Doppler interrogation demonstrates a toand fro-flow into the cavity-flow from the LVOT into the cavity in systole and reverse flow in diastole-and may help depict a rupture of the pseudoaneurysm into adjacent structuresmost frequently the LA, the aorta and the pericardium- . Importantly, when a fistula forms into the LA, it creates an eccentric jet of regurgitation akin to mitral valve regurgitation (MR), which is why a high index of suspicion for MAIVF$\mathrm{P}$ is required in the context of $\mathrm{AV}$ endocarditis and eccentric 
MR jets, in order to identify the supramitral origin of the jet. Fistula to the aorta can be recognized by its more complicated color flow pattern, namely a systolic flow towards both the pseudoaneurysm and the aorta, and a diastolic flow from the pseudoaneurysm to the aorta and then back to the LVOT, that diminishes MAIVF-P pulsatility and results in AR. Pericardial effusion with or without tamponade may ensue in the case of MAIVF-P rupture into the pericardium. ${ }^{4-6)}$

Differential diagnoses include paravalvular/ring abscesses, aneurysms of the sinuses of Valsalva (SOVA) and contained/ not contained aortic root ruptures. Communication between the LVOT and the pseudoaneurysm as well as the pulsatility of the latter, usually help differentiate MAIVF-P from ring/paravalvular abscesses although it must be noted that abscesses can gradually evolve into pseudoaneurysms-as in the illustrated case 2-. A SOVA aneurysm is usually a congenital aortic wall weakness affecting a single or multiple-if acquired-aortic sinuses. Its echocardiographic appearance is marked by sinus dilatation and a windsock deformity of the SOVA that expands during systole, which are not MAIVF-P characteristics. An aneurysm of the SOVA can also rupture into an adjacent cavity. A contained aortic root rupture-usually associated with regional manipulations and periannular hematoma-may be extremely difficult to discriminate from MAIVF-P by echocardiography.

Apart from echocardiography, CT and magnetic resonance imaging are useful assets in determining pseudoaneurysm extent and its relationship with adjacent structures, whereas coronary angiography is essential in ruling out coronary artery compression or significant atherosclerotic disease preoperatively.

\section{COMPLICATIONS}

Gradual pseudoaneurysm enlargement can lead to compression of adjacent structures or rupture into an adjacent cavity. Possible complications include coronary artery-usually the left circumflex-compression causing myocardial ischemia, pulmonary artery compression leading to pulmonary hypertension, fistula formation into the aorta-with prominent $\mathrm{AR}-$ or the LA, rupture into the pericardium-with ensuing cardiac tamponade-, thrombus formation with distal embolization, distortion of the mitral valve giving rise to mitral regurgitation and heart failure-from volume overload-. High risk features predicting MAIVF-P progression and development of complications are endocarditis, large MAIVF-P dimensions ( $>3 \mathrm{~cm}$ ), bicuspid AV or AR, the presence of fistula to the aorta or to a cardiac chamber, the presence of thrombus into the pseudoaneurysmal cavity and compression of adjacent structures. ${ }^{3 / 4)}$

\section{MANAGEMENT}

Complicated, symptomatic or pseudoaneurysms with high risk features should be surgically excised. The surgical procedure is usually complex and may require AVR, aortic root repair and reconstruction of the MAIVF. The natural course of uncomplicated MAIVF-P is largely unknown, since upon diagnosis the majority of patients undergo surgery to treat symptoms or to prevent complications. Both our patients had a clear indication for surgery. Although endocarditis has been assigned as a high risk feature and mitral valve perforation is a serious complication of MAIVF-P, the negative PET scans as well as the presence of moderate mitral valve regurgitation not compromising left ventricular function in our second patient, may be reassuring features. It should be noted that repeat operations impose important risks in patients whose majority have already been operated at least once. Thirty day and midterm mortality are reported to be $19 \%$ and $31 \%$ respectively in a small series of patients who underwent third-time valve surgery. ${ }^{7)}$ On the other hand, there have been reports of uneventful midterm-up to 5 years from diagnosis-follow up of uncomplicated MAIVF-Ps. ${ }^{899)}$ Generally, a watchful waiting approach with serial imaging and referral for operation should complications or rapid expansion ensue, may be valid for asymptomatic patients with high operative risk.

\section{CONCLUSIONS}

MAIVF-P is usually associated with native/prosthetic valve endocarditis or AV surgery. Clinical presentation is related to its size and association with adjacent structures as well as to the presence of complications. Supraventricular or ventricular arrhythmia may ensue as a result of the cavity's vicinity to the LA or its connection with the LVOT. Complex endocarditis with bivalvular insult and mitral valve perforation is another clinical scenario that warrants a high index of clinical suspicion and a low threshold for repeat TEE interrogations. Although the majority of MAIVF-Ps are operated, conservative management with watchful waiting and serial imaging may be a valid option in uncomplicated cases or high risk surgical patients.

\section{SUPPLEMENTARY MOVIE LEGENDS}

Movie 1. Transesophageal echocardiogram (TEE) showing a pulsatile mitral-aortic intervalvular fibrosa pseudoaneurysm. Color flow Doppler interrogation demonstrates a to- and froflow into the pseudoaneurysm (flow from the left ventricular outflow tract into the cavity in systole and reverse flow in diastole).

Movie 2. Serial transesophageal echocardiograms of the aorta in the long axis showing the evolution of the mitral-aortic intervalvular fibrosa pseudoaneurysm (MAIVF-P). (A) A small echodense space is seen at the MAIVF area, indicating the presence of an abscess. (B) A small vegetation protruding from the aortic bioprosthesis into the left ventricle can be now seen. The abscess has started to drain into the left ventricle creating a slot which expands forming a pseudoaneurysm that progressively increases in size $(\mathrm{C})$ two weeks later $(\mathrm{D})$; four weeks later. $(\mathrm{E})$ Color flow Doppler interrogation demonstrates systolic flow from the LVOT into the cavity with reverse diastolic flow.

Movie 3. Serial transesophageal echocardiograms of the aor- 
ta in the short axis plane showing the evolution of the mitralaortic intervalvular fibrosa pseudoaneurysm. (A) An abscess is seen between 9 to 1 o'clock positions around the bioprosthetic valve. The aortic root thickening gradually evolved into a small pseudoaneurysm two weeks later (B) and increased in size in the following four weeks (C).

\section{REFERENCES}

1. Redfearn DP, Hill JD, Keal R, Toff WD, Stafford PJ. Left ventricular dysfunction resulting from frequent unifocal ventricular ectopics with resolution following radiofrequency ablation. Europace 2003;5:247-50.

2. Waldhausen JA, Petry EL, Kurlander GJ. Successful repair of subvalvular annular aneurysm of the left ventricle. N Engl J Med 1966;275: 984-7.

3. Sudhakar S, Sewani A, Agrawal M, Uretsky BF. Pseudoaneurysm of the mitral-aortic intervalvular fibrosa (MAIVF): a comprehensive review. $J$ Am Soc Echocardiogr 2010;23:1009-18; quiz 1112.

4. Afridi I, Apostolidou MA, Saad RM, Zoghbi WA. Pseudoaneurysms of the mitral-aortic intervalvular fibrosa: dynamic characterization using transesophageal echocardiographic and Doppler techniques. J Am Coll Cardiol 1995;25:137-45.

5. Tingleff J, Egeblad H, Gøtzsche CO, Baandrup U, Kristensen BO, Pilegaard H, Pettersson G. Perivalvular cavities in endocarditis: abscesses versus pseudoaneurysms? A transesophageal Doppler echocardiographic study in 118 patients with endocarditis. Am Heart J 1995;130:93-100.

6. Daniel WG, Mügge A, Martin RP, Lindert O, Hausmann D, Nonnast-Daniel B, Laas J, Lichtlen PR. Improvement in the diagnosis of abscesses associated with endocarditis by transesophageal echocardiography. $N$ Engl J Med 1991;324:795-800.

7. Elhmidi Y, Günzinger R, Deutsch MA, Badiu CC, Krane M, Lange R. Outcomes of patients undergoing third-time aortic or mitral valve replacement. J Card Surg 2014;29:8-13.

8. Hasin T, Reisner SA, Agmon Y. Large pseudoaneurysms of the mitralaortic intervalvular fibrosa: long-term natural bistory without surgery in two patients. Eur J Echocardiogr 2011;12:E24.

9. Gin A, Hong H, Rosenblatt A, Black M, Ristow B, Popper R. Pseudoaneurysms of the mitral-aortic intervalvular fibrosa: survival without reoperation. Am Heart J 2011;161:130.e1-5. 